\title{
A novel peripheral biomarker for depression and antidepressant response
}

\author{
Steven D. Targum (iD ${ }^{1,2}{ }^{凶}$, Jeffrey Schappi ${ }^{2,3}$, Athanasia Koutsouris ${ }^{3}$, Runa Bhaumik ${ }^{3}$, Mark H. Rapaport ${ }^{4}$, Natalie Rasgon (iD ${ }^{5}$ and \\ Mark M. Rasenick (D) $2,3,6$ 远
}

This is a U.S. government work and not under copyright protection in the U.S.; foreign copyright protection may apply 2021

\begin{abstract}
In contrast to healthy controls, the heterotrimeric G protein, Gsalpha (Gsa) is ensconced predominantly in lipid rafts in subjects with major depressive disorder (MDD) resulting in impaired stimulation of adenylyl cyclase. In this small proof-of-concept study, we examined the hypothesis that translocation of Gsa from lipid rafts toward a more facile activation of adenylyl cyclase is a biomarker for clinical response to antidepressants. There were 49 subjects with $\mathrm{MDD}$ ( $\mathrm{HamD}_{17}$ score $\geq 15$ ) and 59 healthy controls at the screen visit. The AlphaScreen (PerkinElmer) assay measured both basal activity and prostaglandin E1 (PGE1) stimulation of Gsa-adenylyl cyclase to assess the extent of coupling of Gsa with adenylyl cyclase. At screen, platelet samples obtained from MDD subjects revealed significantly lower PGE1 activation of adenylyl cyclase activity than controls $(p=0.02)$. Subsequently, 19 consenting MDD subjects completed a 6-week open label antidepressant treatment trial. The 11 antidepressant responders (HamD $\mathrm{D}_{17}$ improvement $\geq 50 \%$ from screen) revealed significant increase in PGE1-stimulated adenylyl cyclase compared to non-responders ( $p=0.05)$ with an effect size of 0.83 for the PGE1/Gsa lipid-raft biomarker. PGE1 stimulation increased by $\geq 30 \%$ from screen assessment in eight responders (72.7\%) and two non-responders (25.0\%) [Fisher exact $=0.07$ ] with a positive predictive value for response of $80.0 \%$. In this small, pilot study, increased PGE1 stimulated adenylyl cyclase was associated with antidepressant response in MDD subjects. These data suggest that a simple, high-throughput-capable assay for depression and antidepressant response can be developed. Future studies are needed to evaluate the utility of this biomarker for the treatment of MDD.
\end{abstract}

Molecular Psychiatry (2022) 27:1640-1646; https://doi.org/10.1038/s41380-021-01399-1

\section{INTRODUCTION}

The World Health Organization states that major depressive disorder (MDD) is the most common cause of disability worldwide with a lifetime prevalence close to $20 \%$, and that MDD affects as many as one in ten individuals at any given time [1, 2]. The medical and non-medical costs associated with MDD are estimated at nearly $\$ 300$ billion per year in the United States alone [3]. The current COVID-19 pandemic suggests that depression is a common sequela of those contracting moderate to severe forms of the disease [4]. Although often beneficial, antidepressant treatment is not always effective, may require months to work, and approximately one-third of treated subjects do not achieve remission [5]. Further, the adverse events associated with antidepressants may occur early in the treatment course and contribute to medication non-compliance before the drugs have had a chance to achieve clinical efficacy. Given the substantial medical, economic, and social costs involved with MDD, there is a clear need for a practical and quantitative method to differentiate and optimize treatment options as early as possible. A simple, objective, fast and accurate test for the diagnosis of MDD and prediction of treatment response would abet treatment planning and likely support medication compliance as well.

\section{G proteins/cAMP/CREB/neurotrophin in depression and antidepressant action}

Several studies (vide infra) indicate that chronic antidepressant treatment increases physical coupling between Gsalpha (Gsa) and adenylyl cyclase, resulting in increased cAMP generation. This finding is consistent with the observation that chronic treatment with antidepressants results in long-term increases in cellular CAMP [6]. Consistent with this, depressed subjects show decreased ${ }^{11} \mathrm{C}$ rolipram binding that recovers with successful antidepressant therapy [7]. There exists direct evidence that CAMP signaling may be involved in antidepressant action. O'Donnell and Zhang [8] discuss antidepressant properties of PDE4 inhibitors (and PDE4 knockout in mice). The long-term sequelae of antidepressant treatment include sustained CAMP increases as well as CAMPinduced transcription of growth factor genes [9]. In a recent clinical study, increasing cAMP with inhibitors of phosphodiesterase showed promising antidepressant-adjuvant properties [10]. The initial studies showing that CREB knockout blocks the behavioral response to antidepressants date back 20 years and more recent papers target serotonergic and noradrenergic neurons in achieving this effect [11, 12]. BDNF and TrkB knockout also ablated antidepressant effects in mice [13]. Both humans and mice with the BDNF val66met allele are more vulnerable to stress-

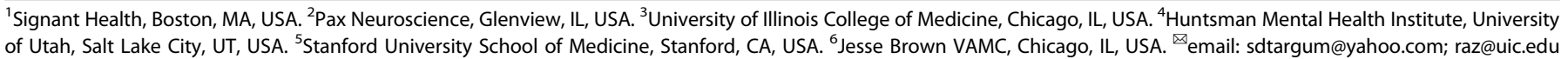


induced anxiety and depression, but this is variable with age and sex $[14,15]$. A polymorphism in the regulatory region of the human BDNF gene, which reduces BDNF expression and release, is also associated with depression [13].

\section{G protein signaling and lipid rafts}

The localization of $G$ proteins to specific membrane domains such as caveolae and lipid rafts has generated interest in these cholesterol and sphingolipid-rich detergent-resistant membrane domains and how they affect $G$ protein targeting and function $[16,17]$. Lipid rafts have variable effects on signaling, as they promote Gqalpha (Gqa) signaling and inhibit Gsa signaling [17-20]. There is a long experimental history of agents that increase "membrane fluidity" increasing agonist- and Gsa-mediated adenylyl cyclase [21, 22]. Gsa stimulates adenylyl cyclase more efficiently outside of lipid rafts and chronic treatment with antidepressants facilitates $G$ protein exodus from those rafts $[19,20,23-27]$. Sublette et al. recently reviewed lipid rafts and the possible roles of these structures in the biology of depression and Kapoor and colleagues have demonstrated direct effects of antidepressants on lipid bilayers [27, 28].

The accumulating evidence as noted above suggests that antidepressants may translocate Gsa from lipid rafts and, in so doing, facilitate the stimulation of adenylyl cyclase by Gsa in the non-raft membrane fraction. These findings suggest that lipid rafts from depressed subjects would show enriched Gsa consistent with diminished CAMP signaling. Donati and colleagues observed enriched Gsa in lipid rafts in both the prefrontal cortex and cerebellum [29]. There was no major change in the total Gsa, and no other $G$ proteins were altered in lipid raft localization in MDD subjects versus controls. More recent post-mortem data showed that the anchors (non-acetylated tubulin) for Gsa in lipid rafts were increased significantly in samples from MDD subjects [30], providing a cellular rationale for the enrichment of Gsa in rafts from depressed subjects. Antidepressant treatment compromised the association between Gsa and tubulin in lipid rafts [26].

Studies in white blood cells [31] and platelets [32,33] suggested the possibility that Gsa stimulated adenylyl cyclase showed results consistent with attenuated CAMP generation in MDD. These findings suggest that a modern adenylyl cyclase assay taken from blood cells might reflect a diagnosis of MDD and antidepressant response and may be adaptable to high throughput screening.

We hypothesize that antidepressant treatment changes Gsa such that it exits from the lipid raft, moves to the non-raft region, and completes the process of neurotransmitter action by stimulating the enzyme, adenylyl cyclase. The objective of the current pilot study of subjects with MDD was to collect preliminary data on the effect of antidepressant treatment on the putative Gsa biomarker.

\section{MATERIALS/SUBJECTS AND METHODS}

The clinical data and platelets for this study came from a 6-week open-label, naturalistic antidepressant trial of participants experiencing an acute major depressive episode to assist the development of a blood-biomarker test for quantitation of depression and prediction of therapeutic response conducted at the Emory University School of Medicine.

\section{Subject selection}

The study recruited depressed subjects with non-psychotic MDD meeting DSM-IV TR criteria for MDD based upon the Structured Clinical Interview for DSM-IV [34-36], and mentally healthy controls. All subjects were male and females between the ages of 30-65 who signed an informed consent approved by an institutional review board to participate in this study. The study was conducted at the Mood and Anxiety Disorders Program research clinic at the Emory University Briarcliff campus between September 2013 and May 2016.
Eligible depressed subjects had a 17-item Hamilton rating scale for depression $\left(\mathrm{HamD}_{17}\right)$ score that was $\geq 15$ at the screen visit [37]. Depressed subjects were excluded from the study if they met criteria for bipolar disorder, a primary psychotic disorder, dementia, substance abuse or dependence within 3 months of the screening visit or presented with a clinically significant suicide risk. The Columbia Suicide Severity Rating Scale was used as part of the assessment of suicidal risk [38]. Eligible depressed subjects had not been taking antidepressant or other psychotropic medications (except for sedatives) for at least 4 weeks prior to the initiation of treatment. Healthy controls had scores $\leq 7$ on the $\mathrm{HamD}_{17}$ and had no history of MDD or dysthymia, and no current psychiatric diagnosis as assessed by the SCID-IV.

\section{Study design}

The study consisted of 2 required visits and an optional third visit for treated MDD subjects. At Visit 1, consenting participants underwent a diagnostic interview by a study psychiatrist who completed the SCID-IV to confirm the presence of MDD and absence of exclusionary diagnoses. In addition, clinical ratings scales were administered by a trained rater and participants completed the Inventory of Depressive Symptomatology Selfreport (IDS-SR $\mathrm{S}_{30}$ ) to assess severity of depressive symptoms over the past week [39]. Vital signs, medical history and physical examination were performed, followed by screening laboratory urine testing (urine drug screen, urinalysis, urine pregnancy test) and phlebotomy for complete metabolic profile, complete blood count, thyroid stimulating hormone test, and for collection of the Gsa blood draw ( $30 \mathrm{ml}$ of whole blood). The sample was collected without regard for fasting status or time of day (usually between 10 A.M. and 3 P.M.), as initial volunteer blood draws for methods development showed that these variables are unrelated to the Gsa content or distribution in platelets.

Visit 2 was scheduled 7 days after Visit 1 and consisted of vital signs, adverse events, and concomitant medications review, and repeat of the Gsa blood draw $(30 \mathrm{ml})$ to assess the test-retest reliability of the Gsa measure over two separate time points. At this visit, MDD participants who desired pharmacotherapy were prescribed an antidepressant medication after consultation with a study psychiatrist. Participants who completed 6 weeks of pharmacotherapy were invited to return for a repeat assessment of their symptoms and to repeat the Gsa $30 \mathrm{ml}$ blood draw to evaluate change in the Gsa measure after treatment.

Visit 3 was scheduled 6 weeks after the initiation of the openlabel antidepressant medication. Visit 3 included all clinical ratings, participant self-ratings, a blood draw of $30 \mathrm{ml}$, vital signs, and a review of concomitant medications and adverse events.

Visits 1 and 3 assessments included clinician ratings of the $\mathrm{HamD}_{17}$ (primary measure), HamD (derived from the HamD ${ }_{17}$ ), Montgomery Asberg depression rating scale (MADRS), Hamilton rating scale for anxiety (Ham-A), the Clinical Global Impression of Severity (CGI-S), and a self-report measure (the Inventory of Depressive Symptoms-self rating form, IDS-SR 30 ) that was completed by the study participant [37, 39-43].

Study participants received $\$ 100$ for completion of Visit $1, \$ 50$ for Visit 2, and \$25 for Visit 3.

\section{Preparation of Gsa biomarker samples}

After each blood draw, blood was separated into red blood cells, white blood cells, and platelet-rich plasma. To ensure blinding, all blood samples were identified by the participant study ID number only.

Each sample was divided into aliquots and frozen at $-80^{\circ} \mathrm{C}$ until further use. Prior to use, platelets were thawed, diluted 1:10 in TEM buffer (10 mM Tris $\mathrm{HCl}, 1 \mathrm{mM} \mathrm{MgCl}, 150 \mathrm{mM} \mathrm{NaCl} 1 \mathrm{mM}$ EDTA pH 7.5, protease inhibitor cocktail, Sigma \# P2714) and adjusted to a concentration of $1 \mu \mathrm{g} / \mu \mathrm{L}$ for the adenylyl cyclase assay. 


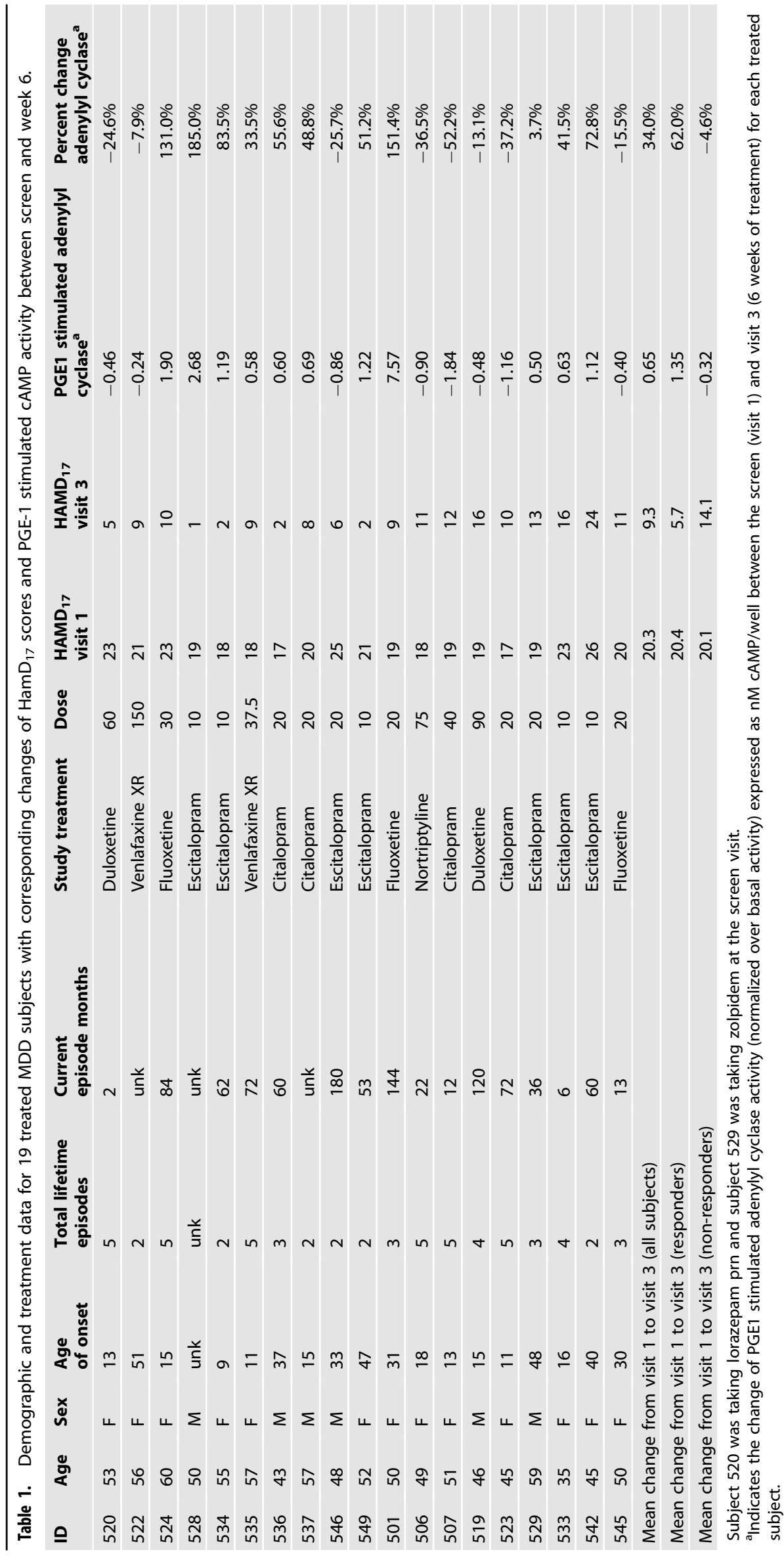




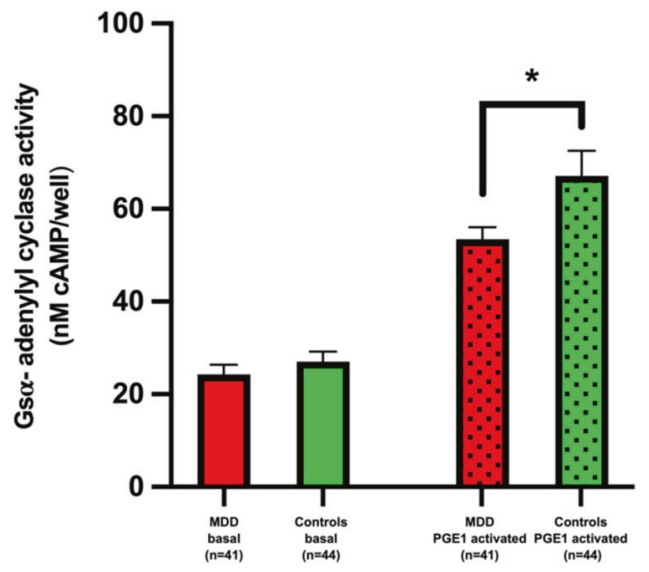

Fig. 1 PGE-1 stimulated CAMP signaling response in platelets is significantly lower in subjects with MDD than healthy controls. Platelets collected from 41 MDD subjects and 44 healthy controls at the screen visit were isolated and assayed for cAMP. Values displayed are mean \pm positive standard error of the mean (SEM). Basal CAMP activity at the screen visit was $24.3 \pm 13.6$ (SD) nM CAMP/ well in the MDD subjects and $27.1 \pm 14.1 \mathrm{nM}$ CAMP/well in the healthy controls: $t=-0.87 ; p=$ ns. *PGE1 stimulated CAMP activity at the screen visit was $53.4 \pm 16.5 \mathrm{nM}$ cAMP/well in the MDD subjects and $67.2 \pm 35.7 \mathrm{nM}$ CAMP/well in the healthy controls: $t=$ $-2.3 ; p=0.02$.

Adenylyl cyclase activity was assayed in a 384 well plate using PerkinElmer's AlphaScreen CAMP assay kit following the manufacturer's directions. Briefly, $2.5 \mu \mathrm{L}$ of platelets was mixed with $1.5 \mu \mathrm{L}$ of stimulation buffer $(1 \mu \mathrm{M}$ Hepes pH 7.5, $500 \mu \mathrm{M}$ IBMX, 0.1\% BSA, 25 $\mathrm{mM} \mathrm{MgCl} 2,375 \mathrm{mM} \mathrm{NaCl}, 250 \mu \mathrm{M}$ ATP, $2.5 \mu \mathrm{M}$ GDP, $2.5 \mathrm{nM}$ GTP in HBSS) in one well of a 384 well plate. Subsequently, $2.5 \mu \mathrm{L}$ of acceptor beads in stimulation buffer were mixed with the platelets and $5 \mu \mathrm{L}$ total volume of cells/beads was added to each well. Adenylyl cyclase activity was measured both without stimulating agent (basal, $5 \mu \mathrm{L}$ stimulation buffer) and in the presence of $10 \mu \mathrm{M}$ prostaglandin E1 (PGE1) in $5 \mu \mathrm{L}$ of stimulation buffer. The 384 well plate was incubated for $30 \mathrm{~min}$ at RT to allow cAMP accumulation. The reaction was stopped by adding $15 \mu \mathrm{L}$ of $1.67 \times$ biotin-cAMP/ Streptavidin Donor Bead Detection Mix. The plate was sealed and kept in the dark overnight. Plates were read on a Molecular Devices SpectraMax i3x plate reader. cAMP produced was calculated from a standard curve run with each assay.

\section{Data analyses}

We compared basal adenylyl cyclase activity and the magnitude of PGE1 stimulated adenylyl cyclase activity in platelet samples obtained from healthy controls and MDD subjects at visit 1 (screen) and at visit 3 from the MDD subjects who participated in the 6-week open label treatment program. We evaluated the changes from visit 1 (screen) to visit 3 (post-treatment) of PGE1 stimulation of adenylyl cyclase activity over basal activity in antidepressant treatment responders and non-responders. Antidepressant treatment response was defined as $\geq 50 \%$ improvement after 6-weeks of treatment from the total HamD 17 score at the screen visit. Statistical analyses included Student's $t$ tests for independent samples and Spearman's correlation coefficient as appropriate.

\section{RESULTS}

There were 49 study participants who met DSM-IV criteria for MDD, and 59 healthy controls seen at the screen visit ranging in age from 30 to 64 years old (Table 1). There were 12 men and 37 women in the MDD group (mean age $=49.1 \pm 7.8$ years) and 18 men and 41 women in the healthy control group (mean age $=$
$43.6 \pm 11.3$ years). At the screen visit the mean $\mathrm{HamD}_{17}$ was $20.5 \pm$ 3.3 in the whole MDD group, the CGI-S was $4.6 \pm 0.6$, and the IDS$\mathrm{SR}_{30}$ was $35.4 \pm 10.9$. The healthy controls had a mean CGI-S of 1.0 \pm 1.0 and IDS-SR 30 of $2.9 \pm 3.0$ at the screen visit.

Platelet samples were available from 41 MDD subjects and 44 healthy controls at the screen visit. There were no significant age, sex, or weight differences between the MDD subjects and healthy controls. The mean basal cAMP activity was $24.3 \pm 13.6 \mathrm{nM}$ cAMP/ well in the MDD subjects and $27.1 \pm 14.15 \mathrm{nM}$ CAMP/well in the healthy controls $(t=-0.87 ; p=\mathrm{ns})$. As shown in Fig. 1 , PGE1 stimulation of adenylyl cyclase activity yielded a significantly lower stimulation response in the MDD subjects than the healthy controls $(t=-2.3 ; p=0.02)$. EC50 for PGE1 was $0.19 \times 10^{-8}$ for healthy controls and $1.9 \times 10^{-9}$ for MDD subjects.

Twenty-five MDD subjects began antidepressant treatment. As per protocol, treated subjects were not required to return for visit 3. Nineteen subjects completed 6 weeks of treatment and had reliable visit 1 and visit 3 clinical data and platelet samples available for analysis. There were 6 men and 13 women in the group of 19 treated MDD subjects who ranged in age from 35 to 60 years (mean age $=50.6 \pm 6.2$ years). The antidepressants prescribed were escitalopram [7], citalopram [4], fluoxetine [3], duloxetine [2], venlafaxine XR [2], and nortriptyline [1]. Eleven treated subjects were antidepressant treatment responders at 6 weeks. There were no significant demographic differences between the treatment responders and non-responders. The mean total $\mathrm{HamD}_{17}$ score at screen was $20.4 \pm 2.5$ (SD) in the treatment responders and $20.1 \pm 5.0$ in the non- responders $(t=$ $0.19 ; p=\mathrm{ns})$. After 6 weeks of treatment, the $\mathrm{HamD}_{17}$ score improved by $14.6 \pm 3.6$ points in the responders and $6.0 \pm 2.3$ in the non-responders $(t=5.87 ; p<0.0001)$. The other clinical metrics tracked with the $\mathrm{HamD}_{17}$ score changes. At 6 weeks, treatment responders had significantly greater improvement than non-responders on the $\mathrm{HamD}_{6}(t=3.06 ; p=0.007)$, MADRS $(t=$ 3.70; $p<0.002)$, and the CGI-S $(t=3.87 ; p=0.05)$ and revealed non- significant trends on the Ham-A ( $t=1.45 ; p=0.16)$, and selfrated $\mathrm{IDS}_{\mathrm{SR}} \mathrm{SR}_{30}(t=1.91 ; p=0.08)$.

Table 1 lists demographic and treatment data and corresponding changes of the total HamD ${ }_{17}$ scores and PGE1 stimulation of adenylyl cyclase activity (normalized over basal activity) from screen (visit 1) to visit 3 for each of the 19 treated subjects. Antidepressant treatment responders had a significant increase in PGE1 stimulated adenylyl cyclase activity at 6 weeks compared to non-responders (Fig. 2). After 6 weeks of antidepressant treatment, PGE1 stimulation of adenylyl cyclase over basal cAMP activity increased $1.35 \pm 2.3$ (SD) nM CAMP/well from the screen visit in the 11 treatment responders, whereas it decreased $-0.32 \pm 1.0 \mathrm{nM}$ cAMP/well in the eight non-responders $(t=2.14 ; p=0.050)$. Fig. 3 displays graphically, the change of PGE1 stimulation of adenylyl cyclase activity normalized over basal activity between visits 1 and 3 . The calculated effect size (Cohen's d) was 0.83 for the PGE1/Gsa lipidraft biomarker. Antidepressant treatment responders revealed a $62.0 \%$ mean increase of PGE1 stimulation from the screen assessment in contrast to a $-4.6 \%$ decrement in the nonresponder cohort. There was a modest correlation between increased cAMP activity and improvement on the total HamD $\mathrm{H}_{17}$ score between visits 1 and 3 in the 19 treated MDD subjects (Spearman's rank-order correlation: $r_{\mathrm{s}}=0.339, t=1.49, p=0.15$ ). PGE1 stimulation of adenylyl cyclase activity increased by at least $30 \%$ from the screen assessment in 8 of the 11 responders $(72.7 \%)$ on contrast to 2 of the 8 non-responders $(25.0 \%)$ in this small group of subjects (Fisher exact $=0.07$ ), and the positive predictive value of PGE1 activation for treatment response was $80.0 \%$.

\section{DISCUSSION}

We examined the relationship of changes in Gsa stimulated adenylyl cyclase activity in MDD subjects participating in a small, 


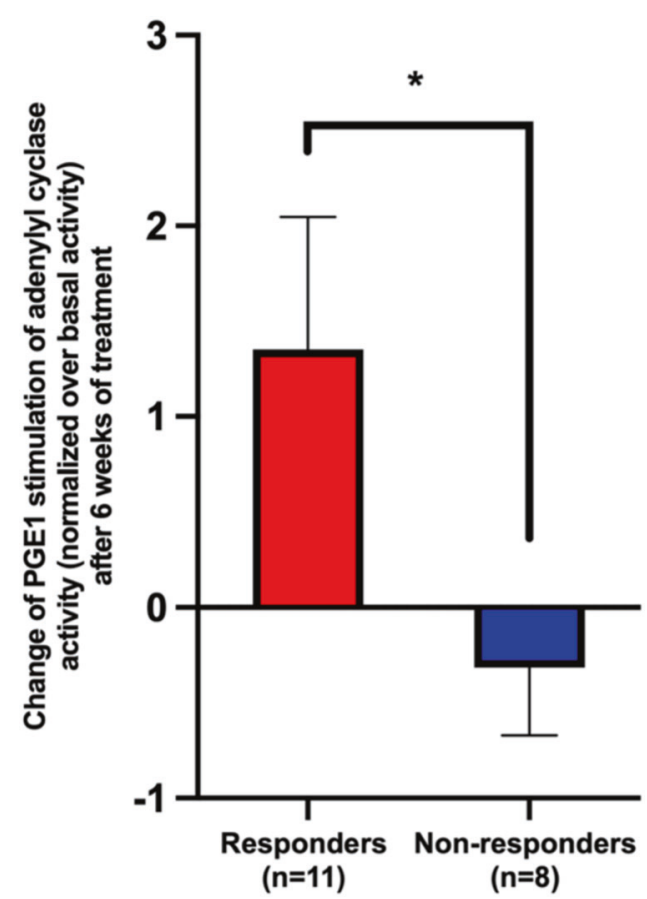

Fig. 2 Change from screen values of PGE-1 stimulated cAMP signaling response in platelets from MDD antidepressant treatment responders and non-responders. Platelets were collected at pre-treatment (visit 1) and post-treatment after 6 weeks of antidepressant treatment (visit 3) from 19 MDD subjects. PGE1 stimulation of adenylyl cyclase activity normalized over basal activity at that visit was assessed and the difference between the screen and 6 weeks scores was calculated. Antidepressant responders were defined as MDD subjects who had total $\mathrm{HamD}_{17}$ score improvement $\geq 50 \%$ between the screen visit and visit 3 . PGE1 change from screen to visit 3 (6 weeks) was $1.35 \pm 2.3$ (SD) in treatment responders compared to $-0.32 \pm 1.0 \mathrm{~nm}$ CAMP/well in non-responders: $t=2.14 ;{ }^{*} p=0.050$. Error bars represent SEM.

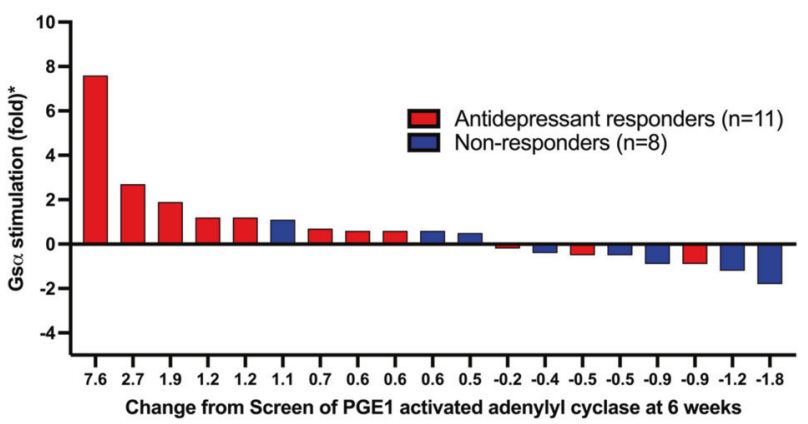

Fig. 3 Antidepressant response to PGE1 stimulated adenylyl cyclase activity: changes in individual subjects from screen visit to 6-weeks of antidepressant treatment. Individual responders and non-responders sort based on pre- vs. post-treatment Gs $\alpha$-activated adenylyl cyclase. Changes in cAMP activity following PGE1 stimulation of adenylyl cyclase between pre-treatment (Visit 1) and posttreatment (Visit 3) for each individual subject appear above.

proof-of-concept 6-week open-label antidepressant treatment trial. First, we found that MDD subjects had significantly lower PGE1 activation of adenylyl cyclase activity in platelet samples than healthy controls at the screen visit $(p=0.02)$. Second, we found that antidepressant treatment responders had a marked increase in PGE1 stimulated adenylyl cyclase after 6-weeks of treatment compared to non-responders $(t=2.14 ; p=0.050)$ with a calculated effect size of 0.83 for the PGE/Gsa lipid-raft biomarker.
PGE1 stimulation of adenylyl cyclase improved by at least $30 \%$ from the screen assessment in 8 of the 11 antidepressant treatment responders (72.7\%) in contrast to 2 of the 8 nonresponders $(25.0 \%)$ in this small study (Fisher exact $=0.07$ ). There was a slight (five-fold) decrease in 5HT potency for controls vs. MDD subjects, consistent with the notion that the relevant biology underlying this biomarker is the coupling between Gsa and adenylyl cyclase dictated by the extent of lipid raft localization of Gsa.

There is post-mortem evidence that suggests that lipid rafts from subjects with MDD are enriched in the heterotrimeric $G$ protein, Gsa consistent with diminished CAMP signaling [29, 30]. Gsa located outside lipid rafts stimulates adenylyl cyclase more efficiently than when it is located within lipid rafts and chronic treatment with antidepressants facilitates $G$ protein exodus from those rafts $[20,23-26,44]$.

Earlier studies that employed more cumbersome CAMP assays suggested that attenuated PGE1 in platelets [32] and impaired $\beta$ adrenergic signaling in white blood cells [31] could be associated with both depression and clinical improvement. It is noteworthy, however, that, in lymphoblasts from depressed subjects we observed increased cAMP signaling which was reversed by antidepressant treatment [45]. Unlike platelets, lymphocytes are negatively affected by CAMP, and attenuated cAMP is "favorable" for the lymphocyte. Caruncho et al. found altered membrane clustering of $5 \mathrm{HT} 2 \mathrm{~A}$ receptors and serotonin transporters in lymphocytes from depressed subjects and attributed this finding to altered cytoskeletal association with these proteins [46]. This finding could be attributed to altered association of these components with lipid rafts.

In preclinical studies, selective serotonin reuptake inhibitors, serotonin-norepinephrine reuptake inhibitors, tricyclic antidepressants, and monoamine oxidase inhibitors all increased Gsa signaling and evoked translocation of Gsa from lipid rafts. Studies in rodents required three weeks of drug treatment while studies in neuronal and glial cells required three days. Extending treatment time in cellular models allowed drug concentrations used to mirror those used in the clinic. Rapid-acting antidepressant drugs such as ketamine had similar effects to traditional antidepressants in the cellular model systems, but on a much more rapid timescale (15 min rather than 3 days) [47]. Thus, we and others have suggested that antidepressants concentrate in lipid rafts and that this constitutes at least some of the basis for their antidepressant effect $[48,49]$. While most antidepressants sort slowly into lipid rafts, ketamine appears do so rapidly $[47,50]$. Fig. 4 presents a model for Gsa sequestration in rafts during depression and subsequent liberation by antidepressants, which we detected as an increase in PGE1-activated adenylyl cyclase. Thus, we hypothesized that the translocation of Gsa from lipid rafts, as reflected by an increase in PGE1 stimulated adenylyl cyclase, might be a biomarker for clinical response to antidepressants.

This was a small pilot study, and the findings must be interpreted with caution. In addition, the study has several limitations that must be noted. First, treatment was open-label and lacked a placebo group, so we could not establish that antidepressants specifically caused the changes in PGE1 stimulated adenylyl cyclase as opposed to non-specific improvements in depression severity. Is the augmented Gsa stimulation of adenylyl cyclase related specifically to antidepressant treatment or simply to clinical improvement? Second, there were no medication adherence measures taken in this study and we cannot be sure if subjects took the prescribed antidepressants for the duration of the 6-week trial, if at all. It is well established that many subjects do not comply with treatment requirements during a clinical trial and that non-adherence can affect the study outcome [51]. Third, the clinical metrics and Gsa stimulation assessments were only done at pre- and post-treatment, with no interim measures to determine the trajectory of the clinical 


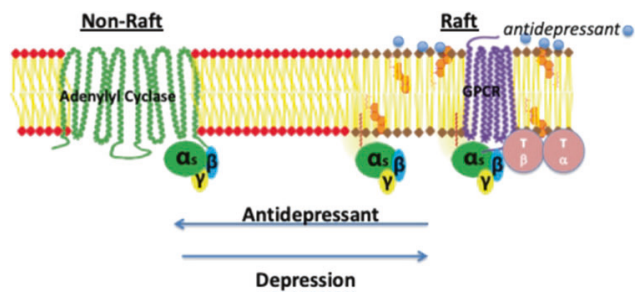

Fig. 4 Effects of depression and antidepressant treatment on Gsa plasma membrane localization. Gs $\alpha$ is normally distributed between non-raft regions of the membrane where it moves freely and promotes neurotransmitter-activated adenylyl cyclase activity and a specialized region of the membrane rich in cholesterol (lipid raft), where the movement/adenylyl cyclase activation of $G s \alpha$ is impaired. During depression, $G s \alpha$ is ensconced in the lipid raft region where it is anchored by the structural protein, tubulin $(T \alpha /$ $T ß)$. Antidepressant treatment changes $G s \alpha$ such that it exits from the raft and moves to the non-raft region where it completes the process of neurotransmitter action by activating the enzyme, adenylyl cyclase.

response or its relationship to increases in Gsa stimulated adenylyl cyclase. Did Gsa translocation/stimulation occur early in the treatment course and is it a harbinger of eventual clinical response? Can this biomarker be used to warrant further treatment in depressed subjects who have not yet responded to ongoing antidepressant treatment or justify changing the treatment regimen? Recently, daily measurement of clinical response via remote ecological momentary assessments (EMA) has been used to examine the mood fluctuation that is an inherent part of MDD and to obtain a more detailed measurement of the clinical trajectory during treatment [52].

Ideally, the methods described herein can form the basis of a quantifiable diagnostic and treatment paradigm. All the limitations noted above can be addressed in a larger, placebocontrolled study that monitors adherence, uses more frequent clinical assessments such as EMA, and includes mid-study biomarker assessments.

Is the magnitude of Gsa stimulation of adenylyl cyclase related to the amount of clinical improvement? Although Fig. 3 suggests that there was a relationship between the magnitude of Gsa stimulation and $\mathrm{HamD}_{17}$ improvement at 6-weeks (Spearman's rank-order correlation was $r_{\mathrm{s}}=0.34$ ), the study was too small to explore this question adequately. As noted above, the clinical response may have been related to the prescribed antidepressant or to placebo responsiveness, and non-response may have been due to antidepressant treatment non-compliance. Further, the study was too small to identify possible moderating factors that might elucidate why the PGE/Gsa lipid-raft biomarker was sensitive for most but not all treatment responders. Clearly, a larger, placebo- controlled trial is warranted to further investigate the potential usefulness of the PGE/Gsa lipid-raft biomarker in MDD. A larger study would allow us to compare the effects of different classes of antidepressants on the PGE/Gsa lipid-raft biomarker. In addition, it would be of interest to explore the usefulness of this biomarker for non-pharmacologic interventions for MDD as well.

In this initial proof-of-concept study, the translocation of Gsa from lipid rafts, as reflected by an increase in PGE1 stimulated adenylyl cyclase, was used as a biomarker reflecting cAMP signaling/lipid-raft status of Gsa, and was associated with antidepressant treatment response in MDD subjects. These data suggest that a simple, high-throughput-capable assay for MDD and antidepressant response can be developed and used to create a platform for personalized medicine for subjects with MDD. Future studies will determine the utility of this biomarker and whether it can anticipate antidepressant response early in treatment and prior to the clinical metrics.

\section{REFERENCES}

1. Malhi GS, Mann JJ. Depression. Lancet. 2018;392:2299-312.

2. Liu $Q$, He H, Yang J, Feng $X$, Zhao F, Lyu J. Changes in the global burden of depression from 1990 to 2017: findings from the Global Burden of Disease study. J Psychiatr Res. 2020;126:134-40.

3. Greenberg PE, Fournier AA, Sisitsky T, Simes M, Berman R, Koenigsberg S, et al. The economic burden of adults with major depressive disorder in the United States (2010 and 2018). PharmacoEconomics. 2021;39:653-65.

4. Huang C, Huang L, Wang Y, Li X, Ren L, Gu X, et al. 6-month consequences of COVID-19 in patients discharged from hospital: a cohort study. Lancet 2021;397:220-32.

5. Trivedi MH, Rush AJ, Wisniewski SR, Nierenberg AA, Warden D, Ritz $L$, et al. Evaluation of outcomes with citalopram for depression using measurementbased care in $\mathrm{STAR}^{*} \mathrm{D}$ : implications for clinical practice. Am J Psychiatry. 2006;163:28-40.

6. Malberg JE, Blendy JA. Antidepressant action: to the nucleus and beyond. Trends Pharm Sci. 2005;26:631-8.

7. Fujita M, Richards EM, Niciu MJ, lonescu DF, Zoghbi SS, Hong J, et al. CAMP signaling in brain is decreased in unmedicated depressed patients and increased by treatment with a selective serotonin reuptake inhibitor. Mol Psychiatry. 2017;22:754-9.

8. O'Donnell JM, Zhang HT. Antidepressant effects of inhibitors of CAMP phosphodiesterase (PDE4). Trends Pharm Sci. 2004;25:158-63.

9. Racagni G, Popoli M. Cellular and molecular mechanisms in the long-term action of antidepressants. Dialogues Clin Neurosci. 2008;10:385-400.

10. El-Haggar SM, Eissa MA, Mostafa TM, El-Attar KS, Abdallah MS. The phosphodiesterase inhibitor pentoxifylline as a novel adjunct to antidepressants in major depressive disorder patients: a proof- of-concept, randomized, double-blind, placebo-controlled trial. Psychother Psychosom. 2018;87:331-9.

11. Blendy JA. The role of CREB in depression and antidepressant treatment. Biol Psychiatry. 2006;59:1144-50.

12. Rafa-Zabłocka K, Kreiner G, Bagińska M, Kuśmierczyk J, Parlato R, Nalepa I. Transgenic mice lacking CREB and CREM in noradrenergic and serotonergic neurons respond differently to common antidepressants on tail suspension test. Sci Rep. 2017;7:13515.

13. Björkholm C, Monteggia LM. BDNF-a key transducer of antidepressant effects. Neuropharmacology. 2016;102:72-79.

14. Hwang JP, Tsai SJ, Hong CJ, Yang CH, Lirng JF, Yang YM. The Val66Met polymorphism of the brain- derived neurotrophic-factor gene is associated with geriatric depression. Neurobiol Aging. 2006;27:1834-7.

15. Verhagen $M$, van der Meij $A$, van Deurzen $P$, Janzing JGE, Arias-Vásquez A, Buitelaar JK, et al. Meta-analysis of the BDNF Val66Met polymorphism in major depressive disorder: effects of gender and ethnicity. Mol Psychiatry. 2010;15:260-71.

16. Li S, Okamoto T, Chun M, Sarguacini M, Casanova JE, Hansen SH, et al. Evidence for a regulated interaction between heterotrimeric $G$ proteins and caveolin. J Biol Chem. 1995;270:15693-701.

17. Allen JA, Halverson-Tamboli R, Rasenick MM. Lipid raft microdomains and neurotransmitter signaling. Nat Rev Neurosci. 2007;8:128-40.

18. Bhatnagar A, Sheffler DJ, Kroeze WK, Compton-Toth B, Roth BL. Caveolin-1 interacts with $5-\mathrm{HT} 2 \mathrm{~A}$ serotonin receptors and profoundly modulates the signaling of selected Galphaq-coupled protein receptors. J Biol Chem. 2004;279:34614-23.

19. Allen JA, Yu JZ, Donati RJ, Rasenick MM. Beta-adrenergic receptor stimulation promotes g\{alpha\}s internalization through lipid rafts: a study in living cells. Mol Pharm. 2005;67:1493-504.

20. Allen JA, Yu J-Z, Dave RH, Bhatnagar J, Roth BL, Rasenick MM. Caveolin-1 and lipid microdomains regulate Gs trafficking and attenuate Gs/adenylyl cyclase signaling. Mol Pharmacol. 2009;76:1082-108.

21. Rimon G, Hanski E, Braun S, Levitzki A. Mode of coupling between hormone receptors and adenylate cyclase elucidated by modulation of membrane fluidity. Nature. 1978;276:394-6.

22. Rasenick MM, Stein $P$, Bitensky $M$. The regulatory subunit of adenylate cyclase interacts with cytoskeletal components. Nature. 1981;294:560-2.

23. Donati RJ, Rasenick MM. Chronic antidepressant treatment prevents accumulation of gsalpha in cholesterol-rich, cytoskeletal-associated, plasma membrane domains (Lipid Rafts). Neuropsychopharmacology. 2005;30:1238-45.

24. Zhang L, Rasenick MM. Chronic treatment with escitalopram but not R-citalopram translocates Galpha s from lipid raft domains and potentiates adenylyl cyclase: a 5-hydroxytryptamine transporter-independent action of this antidepressant compound. J Pharm Exp Ther. 2010;332:977-84.

25. Czysz AH, Schappi J, Rasenick MM. Lateral diffusion of Gas in the plasma membrane is decreased after chronic but not acute antidepressant treatment: Role of lipid raft and non-raft membrane microdomains. Neuropsychopharmacology. 2015;40:766-73. 
26. Singh H, Wray N, Schappi J, Rasenick MM. Disruption of lipid-raft localized Gas/ tubulin complexes by antidepressants: a common feature of HDAC6 inhibitors, SSRI and tTricyclic compounds. Neuropsychopharmacology. 2018;43:1481-91.

27. Liu JJ, Hezghia A, Shaikh SR, Cenido JF, Stark RE, Mann JJ, et al. Regulation of monoamine transporters and receptors by lipid microdomains: implications for depression. Neuropsychopharmacology. 2018;43:2165-79.

28. Kapoor R, Peyear TA, Koeppe RE 2nd, Andersen OS. Antidepressants are modifiers of lipid bilayer properties. J Gen Physiol. 2019;151:342-56.

29. Donati RJ, Dwivedi Y, Roberts RC, Conley RR, Pandey GN, Rasenick MM. Postmortem brain tissue of depressed suicides reveals increased Gs localization in lipid raft domains where it is less likely to activate adenylyl cyclase. J Neurosci. 2008;28:3042-50.

30. Singh H, Chmura J, Bhaumik R, Pandey GN, Rasenick MM. Membrane-associated a-tubulin is less acetylated in postmortem prefrontal cortex from depressed subjects relative to controls: cytoskeletal dynamics, HDAC6 and depression. J Neurosci. 2020;40:4033-41. May 13.

31. Pandey GN, Sudershan $P$, Davis JM. Beta adrenergic receptor function in depression and the effect of antidepressant drugs. Acta Pharm Toxicol. 1985;56 (Suppl 1):S66-S79.

32. Mooney JJ, Schatzberg AF, Cole JO, Kizuka PP, Salomon M, Lerbinger J, et al. Rapid antidepressant response to alprazolam in depressed patients with high catecholamine output and heterologous desensitization of platelet adenylate cyclase. Biol Psychiatry. 1988;23:543-59.

33. Hines LM, Tabakoff B.WHO/ISBRA Study on State and Trait Markers of Alcohol Use and Dependence Investigators Platelet adenylyl cyclase activity: a biological marker for major depression and recent drug use. Biol Psychiatry. 2005;58:955-62.

34. First MB, Spitzer RL, Gibbon M, Williams JBW. Structured Clinical Interview for DSM-IV Axis I Disorders-Patient Edition (SCID-I/P, Version 2.0). Biometrics Research Department, New York State Psychiatric Institute, New York. 1995.

35. American Psychiatric Association. Diagnostic and statistical manual of mental disorders, 4th edition (DSM-IV). Washington DC. 1994.

36. American Psychiatric Association. Diagnostic and statistical manual of mental disorders, 5th edition. Arlington VA: American Psychiatric Press. 2013.

37. Hamilton M. A new depression scale designed to be sensitive to change. $\mathrm{Br} J$ Psychiatry. 1960;134:382-9.

38. Posner K, Brown GK, Stanley B, Brent DA, Yershova KV, Oquendo MA, et al. The Columbia-Suicide Severity Rating Scale: initial validity and internal consistency findings from three multisite studies with adolescents and adults. Am J Psychiatry. 2011;168:1266-77.

39. Rush AJ, Giles DE, Schlesser MA, Fulton $C L$, Weissenburger J, Burns C. The Inventory for Depressive Symptomatology (IDS): preliminary findings. Psychiatry Res. 1986;18:65-87.

40. Hamilton $\mathrm{M}$. The assessment of anxiety states by rating. $\mathrm{Br} \mathrm{J}$ Med Psychol. 1959;32:50-55.

41. Guy W. Early Clinical Drug Evaluation (ECDEU) assessment manual for psychopharmacology. Publication No. 76-338. Rockville, Md: National Institute of Mental Health. 1976.

42. Montgomery S, Asberg M. A new depression scale designed to be sensitive to change. Br J Psychiatry. 1979;134:382-9.

43. Bech P. Rating scales in depression: limitations and pitfalls. Dialogues Clin Neurosci. 2006;8:207-15.

44. Senese NB, Rasenick MM. Antidepressants produce persistent $G a_{s}$-associated signaling changes in lipid rafts after drug withdrawal. Mol Pharm. 2021;100:66-81.

45. Chukaew P, Leow A, Saengsawang W, Rasenick MM. Potential depression and antidepressant-response biomarkers in human lymphoblast cell lines from treatment-responsive and treatment-resistant subjects: roles of SSRIs and omega3 polyunsaturated fatty acids. Mol Psychiatry. 2021;26:2402-14.
46. Caruncho HJ, Rivera-Baltanas T, Romay-Tallon R, Kalynchuk LE, Olivares JM. Patterns of membrane protein clustering in peripheral lymphocytes as predictors of therapeutic outcomes in major depressive disorder. Front Pharm. 2019;10:19047.

47. Wray N, Schappi JM, Singh H, Senese NB, Rasenick MM. NMDA receptor-independent, cAMP- dependent antidepressant actions of ketamine. Mol Psychiatry. 2019;24:1833-43.

48. Eisensamer B, Uhr M, Meyr S, Gimpl G, Deiml T, Rammes G, et al. Antidepressants and antipsychotic drugs colocalize with $5-\mathrm{HT} 3$ receptors in raft-like domains. J Neurosci. 2005;25:10198-206.

49. Erb SJ, Schappi JM, Rasenick MM. Antidepressants accumulate in lipid rafts independent of monoamine transporters to modulate redistribution of the $G$ protein, Gas. J Biol Chem. 2016;291:19725-33.

50. Casarotto PC, Girych M, Fred SM, Kovaleva V, Moliner R, Enkavi G, et al. Antidepressant drugs act by directly binding to TRKB neurotrophin receptors. Cell. 2021;184:1299-313.

51. Dodd S, White IA, Williamson P. Nonadherence to treatment protocol in published randomised controlled trials: a review. Trials. 2012;13:84.

52. Targum SD, Sauder C, Evans M, Saber JN, Harvey PD. Ecological momentary assessment as a measurement tool in depression trials. J Psychiatr Res. 2021;136:256-64.

\section{ACKNOWLEDGEMENTS}

This research was supported by R43MH097370 and R41MH113398 to Pax Neuroscience and VA Merit BX00149 (MMR). MMR is a VA Research Career Scientist BX004475.

\section{AUTHOR CONTRIBUTIONS}

MMR and MHR designed original study. AK and JS collected data. RB, NR, and SDT did statistical analysis. SDT, JS, NR, MHR, and MMR interpreted data. SDT, NR, MHR, JS, and MMR wrote the paper.

\section{COMPETING INTERESTS}

Dr. Targum is an employee of Signant Health and has received vendor grants and/or consulting fees from Acadia Pharmaceuticals Inc., Alkermes Inc., BioXcel Therapeutics Inc., Denovo Biopharma, EMA Wellness LLC, Epiodyne, Frequency Therapeutics, Functional Neuromodulation, Intra Cellular Therapies, Johnson and Johnson PRD, Karuna Therapeutics, Merck Inc., Methylation Sciences Inc., Navitor Pharmaceuticals Inc., and Sunovion Inc. during the past 3 years. Drs Schappi, Bhaumik, Rapaport and Rasgon, and Ms. Koutsouris report no conflicts of interest. Dr. Rasenick is co-founder and chief scientific officer of Pax Neuroscience. In the past three years he has consulted for Otsuka, Inc and has received research support from Lundbeck SA. He is also supported by grants from the Veterans Administration and is a research career scientist of the VA.

\section{ADDITIONAL INFORMATION}

Correspondence and requests for materials should be addressed to Steven D. Targum or Mark M. Rasenick.

Reprints and permission information is available at http://www.nature.com/ reprints

Publisher's note Springer Nature remains neutral with regard to jurisdictional claims in published maps and institutional affiliations. 